\section{WEB WATCH}

- http://www.pancreatica.org

\section{Realistic goals}

Pancreatic cancer has the lowest survival rate of any solid tumour. Although it might be cold comfort to those living with the disease that there is a web site just for them, Pancreatica works well because it's realistic about the chances of a cure and because it isn't overambitious. The site provides only four main resources, but each is carefully thought out and regularly updated. And although it's designed with patients in mind, researchers will find much to enlighten them here.

The most prominent resource is a searchable database of clinical trials. The search page is carefully structured so that the user can select features such as cancer type, trial phase and type of treatment from pulldown menus, as well as searching according to trial locations and whether prior therapy is allowed.

Pancreatic cancer news provides press releases on basic and clinical research. There's also a 'newswire service', providing relevant newspaper articles. There's some overlap in content between this and the news page, and researchers would probably find the former the most useful of the two.

The 'frequently asked questions' section provides clear summaries on 15 questions, spanning diagnosis to end-of-life issues. Controversies, such as the lack of an internationally recognized grading and staging system, are discussed, and hypertext links to other sites are provided.

Pancreatica also provides a list of links and research resources; researchers will find the lists of institutions and charities that fund research into pancreatic cancer valuable. The site provides a sober reminder of why we're doing cancer research, and its producers should be congratulated for their measured approach to a challenging topic.

Cath Brooksbank

\title{
The p53 mafia
}

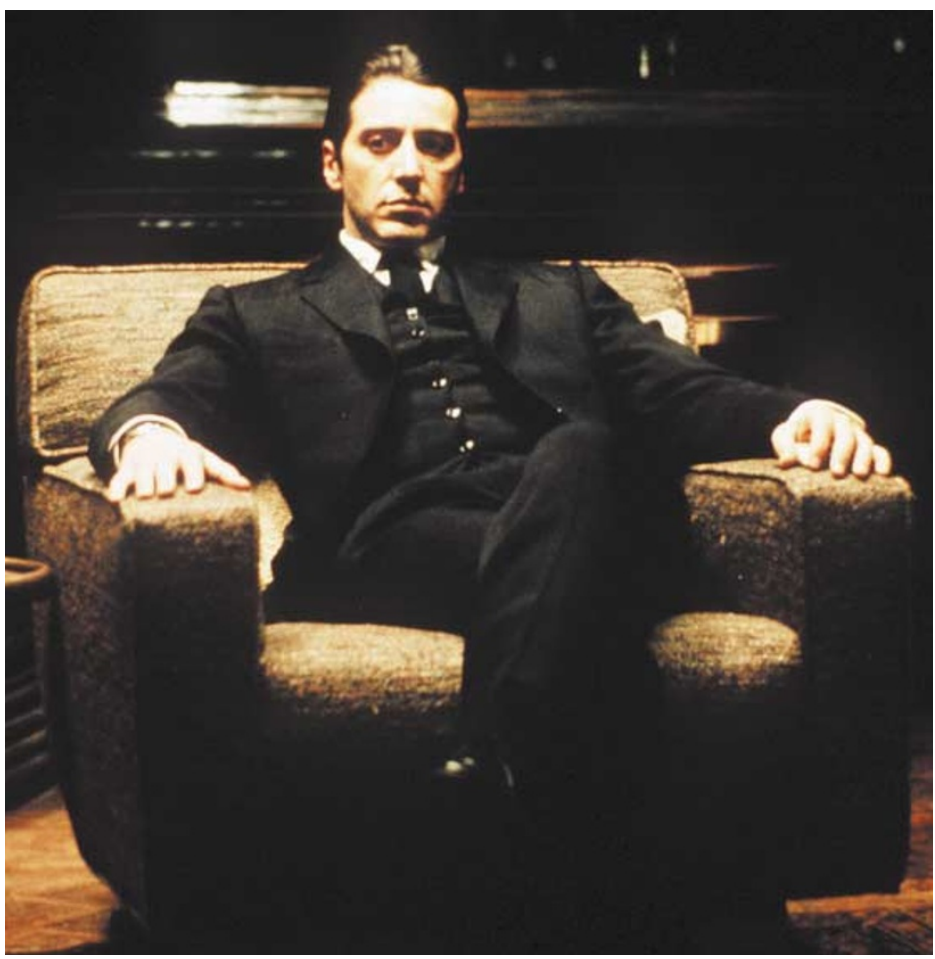

LEUKAEMIA

\section{Common ground}

Scattered evidence has implicated the Notch family - a group of receptors that are involved in cell-fate choices - in the development of T-cell acute lymphoblastic leukaemia (T-ALL). But whether Notch dysregulation is a general rule in these leukaemias, and how it might contribute to neoplasia, has remained a mystery, especially as T-ALL is a diverse disease that is characterized by a range of different cytogenetic changes. Could a single signalling defect unify this mixed bag? Diana Bellavia and colleagues now identify a molecule that seems to collaborate with Notch to cause TALL, and might be a global marker for the disease.

The same group previously generated a mouse model of T-ALL by overexpressing a truncated, constitutively active Notch3 mutant (Notch3-IC) in thymocytes (T-cell precursors). The leukaemic cells expressed $\mathrm{pT} \alpha$, a component of the pre-Tcell receptor that enhances thymocyte survival and proliferation in the absence of an antigenic stimulus. Might human T-ALL cells also express pT $\alpha$ ? Reverse transcriptase polymerase chain reaction and northern blot analysis of $30 \mathrm{~T}$-ALL cases revealed that Notch 3 and $\mathrm{pT} \alpha$ were expressed in all cases examined. HES1, a transcription factor that is downstream of Notch signalling, was also expressed in T-ALL cells. By contrast, normal peripheral T cells expressed none of these transcripts. Notch 3 and $p T \alpha$ expression also decreased to undetectable levels in T-ALL that was in remission.
At the heart of a report in Nature lies a tale of drugs and violent death. The leading role in this story goes to $\mathrm{p} 53$, which orders the apoptotic execution of cells in response to DNA-damaging agents such as doxorubicin. But as Elsa Flores, Tyler Jacks and co-workers now show, this is a family affair - for p53 needs its close relatives p63 and p73 to carry out the task.

The structural and functional similarities between $\mathrm{p} 53$ and its relatives led Jacks and colleagues to ask whether $\mathrm{p} 63$ and $\mathrm{p} 73$ are involved in the p53-dependent response to DNAdamaging agents. They studied this using mouse embryo fibroblasts (MEFs) that had been sensitized to undergo apoptosis by expression of the E1A oncogene and were deficient in various p53 family members.

As expected, after treatment with doxorubicin, the $\operatorname{Tr} p 53^{--}$MEFs were resistant to apoptotic death. The $\operatorname{Trp} 63^{-/-}$and $\operatorname{Trp} 73^{-/-}$cells showed only a partial resistance to apoptosis, but MEFs that lacked both of these genes were as resistant to apoptosis as were the $\operatorname{Tr} p 53^{-/-}$cells. Moreover, cells that lacked $\mathrm{p} 53 / \mathrm{p} 63$ or p53/p73 were more resistant than MEFs that

So is $\mathrm{pT} \alpha$ expression necessary for lymphomagenesis? To investigate this, the authors crossed their Notch3-IC mice with $\mathrm{pT} \alpha^{-1-}$ mice. Notch3-IC mice with one or two copies of $\mathrm{pT} \alpha$ all developed Tcell tumours, whereas only $10 \%$ of Notch3-IC, $\mathrm{pT}^{-/-}$mice developed tumours. This was not due to an inability of $\mathrm{p} T \alpha^{-1-}$ thymocytes to develop properly, as the number of mature peripheral $\mathrm{T}$ cells was almost normal in these mice. So expression of Notch 3 and $p$ T $\alpha$ seems to be a common thread in all cases of T-ALL, regardless of developmental stage or karyotype. Whether Notch 3 provides the signal that sustains $\mathrm{pT} \alpha$ expression is an intriguing question for the future. Cath Brooksbank

(2) References and links ORIGINAL RESEARCH PAPER Bellavia, D. et al. Combined expression of pT $\alpha$ and Notch 3 in T cell leukemia identifies the requirement of preTCR for leukemogenesis. Proc. Natl Acad. Sci. USA 99, 3788-3793 (2002) FURTHER READING Bellavia, D. et al. Constitutive activation of NF- $\mathrm{kB}$ and T-cell leukemia/lymphoma in Notch3 transgenic mice. EMBO J. 19, 3337-3348 (2000) 
were deficient in $\mathrm{p} 53$ alone, and this indicated that $\mathrm{p} 63$ and $\mathrm{p} 73$ might act with $\mathrm{p} 53$ - or in a parallel pathway - to induce apoptosis after DNA damage. The authors confirmed these results in an in vivo system the developing nervous system of day-13.5 mouse embryos, in which p53 has been shown to be important for $\gamma$-irradiation-induced apoptosis.

Although other mechanisms of p53-dependent apoptosis have been proposed, there is a general consensus that $\mathrm{p} 53$ acts in this process by the initiation of downstream target genes. So, Jacks and colleagues studied the induction of p53 target genes in MEFs that lacked the various p53 family members. The induction of some targets, such as Cdknla (which encodes p21) and $M d m 2$, was no different in wild-type cells than in cells that lacked $\mathrm{p} 63, \mathrm{p} 73$ or $\mathrm{p} 63 / \mathrm{p} 73$. But other genes - Bax and Perp, for example - were not expressed in MEFs that lacked p63 and p73. Notably, Bax and Perp are linked to apoptotic responses.

The authors had shown that both p53 and p63 are enriched in the nuclei of MEFs after DNA damage, so they tested whether these proteins can bind the promoters of p53 target genes. Both proteins associated with the Cdkn1a, Mdm2, Bax and Perp promoters in wild-type MEFs. However, in cells that lacked p63 and p73, p53 could no longer associate with the $B a x$ or Perp promoters in response to DNA damage. Moreover, in p53-deficient MEFs, p63 was found specifically at the Bax and Perp promoters.

These results indicate a model in which $\mathrm{p} 63$ and $\mathrm{p} 73$ regulate the ability of p53 to bind at certain promoters after its induction in response to DNA damage. And, as the authors speculate, they might "portend a greater role for these proteins in tumour suppression and chemosensitivity". Alison Mitchell Editor, Nature Reviews Molecular Cell Biology

(2) References and links ORIGINAL RESEARCH PAPER Flores, E. R. et al. p63 and p73 are required for p53-dependent apoptosis in response to DNA damage. Nature 416, 560-564 (2002)

FURTHER READING Yang, A. \& McKeon, F. p63 and p73: p53 mimics, menaces and more. Nature Rev. Mol. Cell Biol. 1, 199-207 (2000) WEB SITE

Tyler Jacks' lab: http://web.mit.edu/biology/ www/facultyareas/facresearch/jacks.shtml

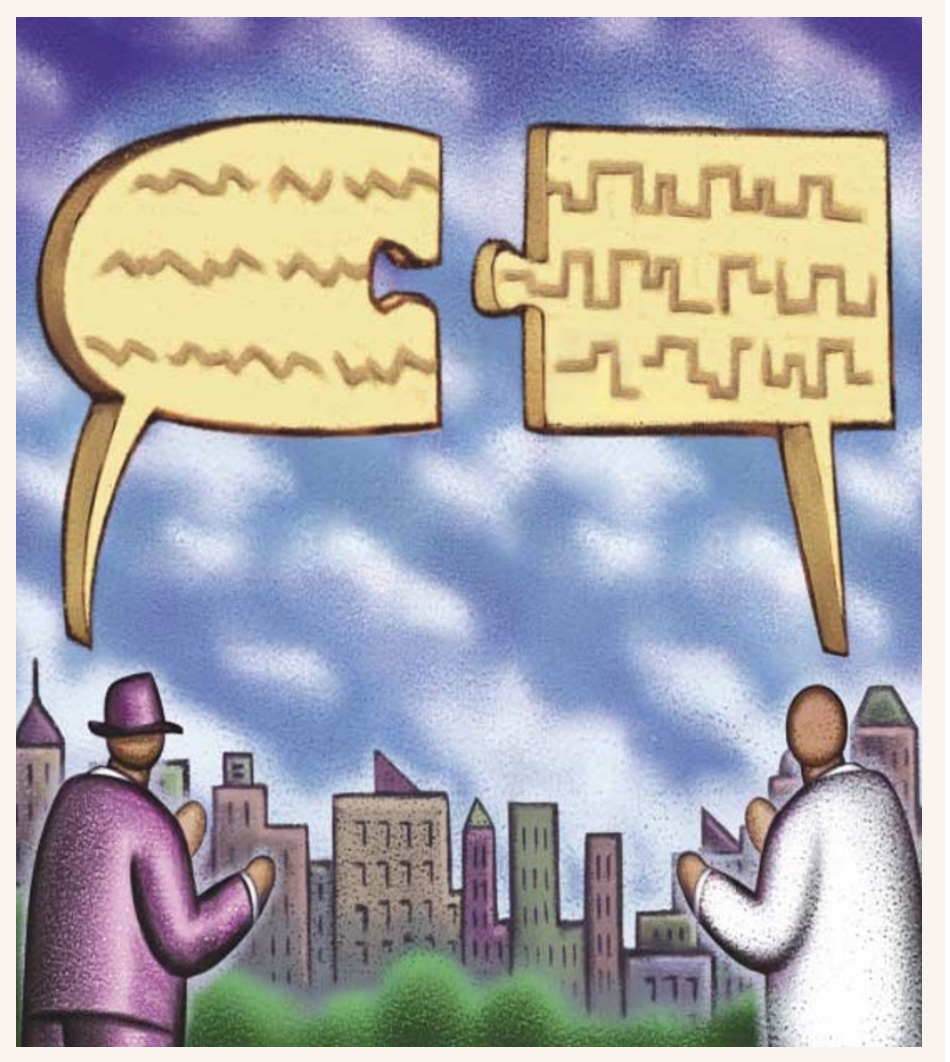

\section{IN BRIEF}

\section{TUMOUR SUPPRESSORS}

\section{HLTF gene silencing in human colon cancer.}

Moinova, R. et al. Proc. Natl Acad. Sci. USA 99, 4562-4567 (2002)

Expression of helicase-like transcription factor (HLTF) - a member of the SWI/SNF family of chromatin-remodelling enzymes, which have been shown to be disrupted in cancer - is lost in $\sim 30 \%$ of colon cancer cell lines. This loss is accompanied by promoter methylation, and is restored following addition of 5-azacytidine, a demethylating agent. Interestingly, the HLTF promoter is not methylated in breast or lung cancer, so HLTF might specifically suppress colon cancer. Consistent with this, its transfection into HLTF-deficient cell lines suppresses colony growth.

\section{P53 REGULATION}

\section{Deubiquitination of p53 by HAUSP is an important} pathway for p53 stabilization.

Li, M. et al. Nature 416, 648-653 (2002)

To function as a tumour suppressor, p53 must first be freed from the clutches of MDM2, a ubiquitin ligase that targets p53 for degradation. Li et al. have used mass spectrometry to identify p53interacting proteins, and have discovered herpesvirus-associated ubiquitin-specific protease (HAUSP). HAUSP can deubiquitylate — and therefore stabilize - p53, even in the presence of MDM2. Accumulation of $\mathrm{p} 53$ results in growth arrest and apoptosis. HAUSP might therefore act as a tumour suppressor, by stabilizing $\mathrm{p} 53$.

\section{MOUSE MODELS}

Nf2 gene inactivation in arachnoidal cells is rate limiting for meningioma development in the mouse.

Kalamarides, M. et al. Genes Dev. 16, 1060-1065 (2002)

Tumours of the membranes that cover the brain, known as meningiomas, are a common affliction of people with neurofibromatosis type 2 , which is caused by inactivation of the $\mathrm{NF} 2$ gene. However, $\mathrm{Nf2^{-- }}$ mice don't develop meningiomas. Michel Kalamarides and colleagues have developed the first genetically modified mouse model of meningioma by knocking out $N f 2$ in a small number of meningeal cells.

\section{THERAPEUTICS}

ErbB2 is essential in the prevention of dilated cardiomyopathy.

Crone, S. et al. Nature Med. 8, 459-465 (2002)

Herceptin (trastuzumab) - a humanized monoclonal antibody specific for the extracellular domain of the receptor tyrosine kinase ERBB2 - is used to treat breast cancers that overexpress ERBB2. Some patients who take this drug, however, experience cardiac dysfunction. To investigate the role of ErbB2 in the adult heart, mice with a ventricular-restricted deletion of ErbB2 were generated. These mice developed many features of dilated cardiomyopathy, indicating that ErbB2 function is required for normal cardiomyocyte function. Strategies to prevent cardiac dysfunction must therefore be developed for patients who take Herceptin. 